\title{
Resultados en el tratamiento quirúrgico del cáncer de colon por vía laparoscópica. Análisis retrospectivo
}

\author{
Nicolás Avellaneda \\ Servicio de Cirugía General y Unidad de Patología Orificial, Centro de Educación Médica e Investigaciones Clínicas \\ "Norberto Quirno" (CEMIC). CABA, Argentina.
}

\begin{abstract}
RESUMEN
Introducción: Los tumores de colon representan una patología prevalente a nivel mundial y la cirugía es de elección para su tratamiento. El advenimiento del abordaje laparoscópico ha sido asociado a mejores resultados postoperatorios. Objetivo: Realizar un estudio comparativo de pacientes operados por cáncer de colon utilizando la vía laparoscópica y convencional.

Materiales y métodos: Se realizó un análisis retrospectivo de todos los pacientes sometidos a cirugía por patología maligna colónica en un hospital, durante un período de 62 meses, comparando aquellos que fueron abordados de forma convencional con aquellos operados por vía laparoscópica. Se incluyeron todos los datos inherentes a la información preoperatoria de los pacientes, características de la técnica utilizada, morbilidad y mortalidad del procedimiento y estadio tumoral.

Resultados: Durante el período evaluado, se realizaron 544 cirugías por patología colorrectal, de las cuales 191 cumplieron con los criterios de inclusión: 41 en el grupo convencional y 150 en el laparoscópico. No hubo diferencias entre grupos en cuanto a edad y comorbilidades, y la cohorte convencional presentó mayor incidencia de cirugías previas. El tiempo operatorio promedio fue de 141,28 minutos en el grupo laparoscópico (DS = 51,65) y de 176,34 minutos en el convencional (DS = 85,02). La morbilidad fue de 53,66 \% y $28 \%(p=0,002)$, la mortalidad fue de $4,88 \%$ y $0 \%(p=0,007)$, y el tiempo de internación fue de 13,04 días ( $\mathrm{DS}=10,10)$ y de 6,95 días $(\mathrm{DS}=4,44)$ para el abordaje convencional y laparoscópico, respectivamente.

Conclusiones: Al igual que otras series, este grupo presenta diferencias significativas a favor del abordaje laparoscópico de los pacientes con cáncer de colon, con menor tiempo operatorio, menor tiempo de internación y menor tasa de complicaciones.
\end{abstract}

Palabras claves: Colon; Cáncer; Laparoscopia; Convencional; Morbilidad; Complicaciones

\begin{abstract}
Introduction: Colon tumors represent a prevalent disease worldwide and is required for their treatment. The advent of the laparoscopic approach has been associated with better postoperative results.

Objective: To compare colon cancer patients treated by laparoscopic and conventional approach.

Material and methods: A retrospective analysis of all patients undergoing surgery for malignant colonic tumors in our hospital during a period of 62 months was performed. Patients approached conventionally were compared with those operated on by laparoscopy. All the preoperative data of patients, as well as the characteristics of the technique used, the morbidity and mortality of the procedure and the tumor stage were included.

Results: During the evaluated period, 544 surgeries were performed for colorectal conditions, of which 191 met the inclusion criteria: 41 in the conventional group and 150 in the laparoscopic group. There were no differences between groups in terms of age and comorbidities, although the conventional cohort had a higher incidence of previous surgical procedures. The mean operative time was $141.28 \pm 51.65$ minutes in the laparoscopic group and $176.34 \pm 85.02$ minutes in the conventional group. Morbidity was 53.66 vs. $28 \%(p=0.002)$, mortality 4.88 vs. $0 \%(p=0.007)$, and hospital stay $13.04 \pm 10,10 \mathrm{vs.} 6.95 \pm$ 4.44 days for the conventional and laparoscopic approach, respectively.

Conclusions: As other series, this study showed significant differences that favor the laparoscopic approach for patients with colon cancer, with shorter operative time, shorter length of stay and lower rate of complications.
\end{abstract}

Key words: Colon; Cancer; Laparoscopy; Conventional; Morbidity; Complications

\section{INTRODUCCIÓN}

La patología neoplásica colorrectal es la tercera en incidencia en ambos sexos a nivel mundial (tercera en hombres, segunda en mujeres) y, a su vez, es la segunda causa de muerte por cáncer. ${ }^{1}$ Se han logrado grandes avances en el diagnóstico temprano de esta enfermedad gracias

Los autores declaran no tener conflicto de intereses.

Nicolás Avellaneda

n.avellaneda86@gmail.com

Recibido: Julio de 2020. Aceptado: Septiembre de 2020 al desarrollo de estrategias poblacionales de pesquisa. ${ }^{2}$ Sin embargo, el desarrollo de este tipo de patología en personas más jóvenes es una tendencia que se observa cada vez más frecuentemente. ${ }^{2}$

El tratamiento quirúrgico continúa siendo de elección para los tumores que se presentan en cualquier segmento del colon. ${ }^{2}$ A principios de 1990, comenzaron a aparecer las primeras publicaciones sobre pacientes operados por vía laparoscópica por patología colónica, ${ }^{3-5}$ representando un cambio de paradigma en el abordaje de este tipo de pacientes. Si bien originalmente recibió algunas críti- 
cas cuestionando la factibilidad oncológica del procedimiento y la presencia de metástasis en orificios de puertos de trabajo, ${ }^{6,7}$ actualmente la vía laparoscópica ha sido propuesta como el estándar de abordaje para el tratamiento del cáncer de colon. ${ }^{8}$

Numerosos estudios han probado los beneficios de la cirugía mínimamente invasiva desde su advenimiento hasta ahora. ${ }^{9-12}$ Sin embargo, este abordaje no ha sido adoptado universalmente, llegando solo al $50 \%$ de los procedimientos operados por esta vía en países desarro1lados. ${ }^{13,14}$ A nivel nacional, un relevamiento encabezado por Patrón Uriburu y colaboradores, en el año 2011, ${ }^{15}$ determinó que solo un $23 \%$ de los cirujanos colorrectales realizaban procedimientos por vía laparoscópica.

El objetivo de este trabajo es presentar la experiencia inicial en colectomías laparoscópicas en un centro de salud y comparar dicho abordaje con aquellos pacientes operados por vía convencional.

\section{MATERIALES Y MÉTODOS}

Se incluyeron en el presente estudio todos aquellos pacientes operados por patología neoplásica del colon en el Hospital Universitario CEMIC (CABA, Argentina), durante el periodo comprendido entre enero de 2015 y marzo de 2020. Se tomaron en cuenta todos aquellos operados de forma programada y se dividieron en dos grupos: abordados por vía convencional y por vía laparoscópica. E1 gráfico 1 desarrolla el proceso de selección de pacientes. Fueron excluidos todos aquellos pacientes operados de urgencia o por patología no neoplásica.

La selección de la técnica fue hecha en base a la experiencia del cirujano. En cuanto al abordaje laparoscópico, todas las cirugías fueron realizadas o supervisadas por un cirujano con experiencia en este tipo de procedimiento.

Se confeccionó una base de datos que incluía:

Información preoperatoria: datos filiatorios, edad, sexo, localización del tumor, comorbilidades, antecedente de cáncer de colon, antecedentes de cirugía abdominal (definido como cualquier tipo de procedimiento que implicara el acceso a la cavidad abdominal por cualquiera de ambos abordajes).

Información de la cirugía y de la internación: Tiempo operatorio, cirugía abdominal asociada, días de internación, complicaciones postoperatorias (estratificadas según clasificación de Dindo-Clavien), ${ }^{16}$ incidencia de dehiscencia anastomótica y clasificación de la misma (siendo menor aquellas clasificadas como A y B por el International Study Group of Rectal Cancer, y mayor aquellas clasificadas como C). ${ }^{17}$

Información de seguimiento: Informe anatomopatoló-

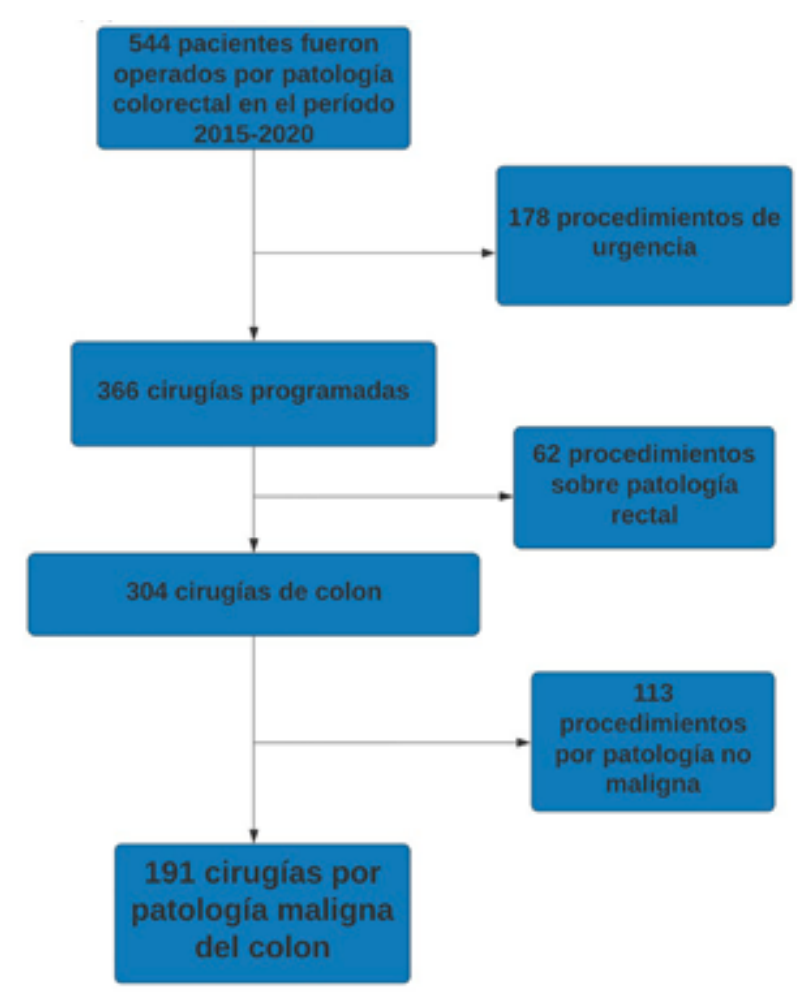

Cuadro 1: Proceso de selección de pacientes.

gico (características histológicas, estadío según clasificación de la American Joint Committee on Cancer). ${ }^{18}$

\section{Descripción técnica quirúrgica}

- Colectomía derecha laparoscópica: Se utilizan tres puertos, dos de $10 \mathrm{~mm}$. En región umbilical y flanco izquierdo y uno de $5 \mathrm{~mm}$. A nivel suprapúbico. Abordaje desde medial hacia lateral y se realiza anastomosis extracorpórea latero-lateral con doble sutura mecánica lineal cortante.

- Colectomía izquierda laparoscópica: Se utilizan tres puertos, dos de $10 \mathrm{~mm}$. A nivel umbilical y en flanco derecho y un puerto de $10 \mathrm{~mm}$. Colocado en fosa ilíaca derecha. Abordaje desde medial hacia lateral, anastomosis intracorpórea colorrectal término-terminal con sutura mecánica circular.

- Abordaje laparoscópico de tumores de colon transverso: Para aquellos ubicados en la vertiente derecha del colon transverso, se realiza una colectomía derecha laparoscópica ampliada, siguiendo los principios ya descriptos. Los tumores adyacentes al ángulo esplénico del colon son tratados realizando un abordaje medial anastomosis colocolónica latero-terminal con sutura mecánica circular.

- Colectomía derecha e izquierda convencional: Laparotomía mediana supra-infra umbilical y abordaje de lateral a medial. Mismos principios para la anastomosis que cirugía laparoscópica. 
TABLA 1: CARACTERÍSTICAS DE LOS PACIENTES

\begin{tabular}{|c|c|c|c|}
\hline Variable & $\begin{array}{l}\text { Cirugía } \\
\text { convencional } \\
\mathrm{n}(\%)\end{array}$ & $\begin{array}{l}\text { Cirugía } \\
\text { laparoscópica } \\
\mathrm{n}(\%)\end{array}$ & $\begin{array}{l}\text { Valor } \\
\text { de } p\end{array}$ \\
\hline $\mathrm{N}$ & $41(100)$ & $150(100)$ & \\
\hline Género femenino & $20(49)$ & $69(46)$ & 0,75 \\
\hline $\begin{array}{l}\text { Edad promedio. } \\
\text { años (rango) }\end{array}$ & $\begin{array}{l}69,10 \\
(37-91)\end{array}$ & $\begin{array}{c}67,92 \\
(28-90)\end{array}$ & 0,30 \\
\hline \multicolumn{4}{|l|}{ Comorbilidades } \\
\hline Hipertensión arterial & $23(56,1)$ & $74(49,3)$ & 0,44 \\
\hline Diabetes II & $7(17,1)$ & $18(12)$ & 0,39 \\
\hline Dislipemia & $18(43,9)$ & $51(34)$ & 0,24 \\
\hline Tabaquismo & $6(14,6)$ & $17(11,3)$ & 0,28 \\
\hline $\begin{array}{l}\text { Enfermedad } \\
\text { pulmonar obstructiva } \\
\text { crónica }\end{array}$ & $5(12,2)$ & $8(5,3)$ & 0,12 \\
\hline $\begin{array}{l}\text { Enfermedad renal } \\
\text { crónica }\end{array}$ & $2(4,9)$ & $4(8)$ & 0,47 \\
\hline \multicolumn{4}{|l|}{ Ubicación } \\
\hline Colon derecho & $19(46,3)$ & $79(52,7)$ & 0,36 \\
\hline Colon transverso & $12(29,3)$ & $21(14)$ & 0,02 \\
\hline Colon izquierdo & $8(19,5)$ & $47(31,3)$ & 0,62 \\
\hline $\begin{array}{l}\text { Afectación de más } \\
\text { de un segmento }\end{array}$ & $2(4,9)$ & $3(2)$ & 0,31 \\
\hline $\begin{array}{l}\text { Cirugías abdominales } \\
\text { previas }\end{array}$ & $31(82,9)$ & $83(55,3)$ & 0,02 \\
\hline
\end{tabular}

Aclaraciones: \%: porcentaje, N: número total de pacientes.

\section{RESULTADOS}

Ciento noventa y un pacientes fueron incluidos en el presente estudio. La media de edad global fue de $68,{ }^{17}$ años. La tabla 1 resume las características preoperatorias de los pacientes, de los cuales 41 fueron abordados por vía convencional $(21,5 \%)$ y 150 por vía laparoscópica $(78,5 \%)$, con requerimientos de conversión en el $10 \%$ de estos casos (15 pacientes). No hubo diferencias significativas en cuanto a la edad, sexo y comorbilidades entre ambos grupos.

Se encontraron diferencias significativas en el porcentaje de tumores localizados en el colon transverso $(24,4$ $\%$ para el grupo convencional y $11,3 \%$ para el laparoscópico, $p=0,03)$. También hubo diferencias en el antecedente de cirugías abdominales previas, siendo este mayor en el grupo convencional $(82,9 \%$ vs. $55,3 \%, \mathrm{p}=$ $0,02)$.

La tabla 2 esquematiza los datos operatorios de los pacientes.

Se encontraron diferencias en el abordaje de los pacientes que presentaban tumores de colon transverso, siendo mayor el número de procedimientos por vía convencional en este grupo $(26,8 \%$ vs. $12,7 \%, p=0,03)$. No hubo diferencias en la vía de abordaje para tumores localiza-
TABLA 2: DATOS OPERATORIOS DE LOS PACIENTES

\begin{tabular}{|c|c|c|c|}
\hline Variable & $\begin{array}{l}\text { Cirugía } \\
\text { convencional } \\
n(\%)\end{array}$ & $\begin{array}{l}\text { Cirugía } \\
\text { laparoscópica } \\
\mathrm{n}(\%)\end{array}$ & $\begin{array}{l}\text { Valor } \\
\text { de } p\end{array}$ \\
\hline $\mathrm{N}$ & $41(100)$ & 150 (100) & \\
\hline \multicolumn{4}{|l|}{ Tipo de cirugía } \\
\hline $\begin{array}{l}\text { Colectomía } \\
\text { derecha }\end{array}$ & $16(39)$ & $75(50)$ & 0,21 \\
\hline $\begin{array}{l}\text { Colectomía } \\
\text { derecha } \\
\text { ampliada }\end{array}$ & $4(9,8)$ & $7(4,7)$ & 0,21 \\
\hline $\begin{array}{l}\text { Colectomía } \\
\text { segmentaria }\end{array}$ & $11(26,8)$ & $19(12,7)$ & 0,03 \\
\hline $\begin{array}{l}\text { Colectomía } \\
\text { izquierda }\end{array}$ & $8(19,5)$ & $46(30,7)$ & 0,16 \\
\hline $\begin{array}{l}\text { Colectomía } \\
\text { total }\end{array}$ & $2(4,9)$ & $3(2)$ & 0,31 \\
\hline $\begin{array}{l}\text { Cirugía } \\
\text { asociada }\end{array}$ & $16(39)$ & $10(6,7)$ & 0,001 \\
\hline $\begin{array}{l}\text { Tiempo } \\
\text { operatorio } \\
\text { (minutos, rango) }\end{array}$ & $\begin{array}{l}176,34(60- \\
460)\end{array}$ & $141,28(60-325)$ & 0,0006 \\
\hline $\begin{array}{l}\text { Anastomosis } \\
\text { primaria }\end{array}$ & $36(87,8)$ & $146(97,3)$ & 0,01 \\
\hline $\begin{array}{l}\text { Cuidados } \\
\text { intensivos en el } \\
\text { post operatorio }\end{array}$ & $4(9,7)$ & 0 & 0,001 \\
\hline
\end{tabular}

TABLA 3: DATOS POSTOPERATORIOS

\begin{tabular}{|c|c|c|c|}
\hline Variable & $\begin{array}{l}\text { Cirugía } \\
\text { convencional } \\
n(\%)\end{array}$ & $\begin{array}{l}\text { Cirugía } \\
\text { laparoscópica } \\
\mathrm{n}(\%)\end{array}$ & $\begin{array}{l}\text { Valor } \\
\text { de p }\end{array}$ \\
\hline $\mathrm{N}$ & $41(100)$ & $150(100)$ & \\
\hline $\begin{array}{l}\text { Estadía hospitalaria } \\
\text { (rango), días }\end{array}$ & $13,3(3-58)$ & $6,9(3-28)$ & 0,0001 \\
\hline Complicaciones & $22(53,6)$ & $42(28)$ & 0,002 \\
\hline \multicolumn{4}{|c|}{ Complicaciones Clasificación Dindo-Clavien } \\
\hline I & $1(2,4)$ & $2(1,3)$ & 0,61 \\
\hline II & $9(22)$ & $22(14,7)$ & 0,27 \\
\hline IIIA & $2(4,9)$ & $3(2)$ & 0,31 \\
\hline IIIB & $5(12,2)$ & $13(8,7)$ & 0,49 \\
\hline IVA & $2(4,9)$ & $1(0,7)$ & 0,06 \\
\hline IVB & $1(2,44)$ & 0 & 0,06 \\
\hline $\begin{array}{l}\text { Infección del sitio } \\
\text { quirúrgico }\end{array}$ & $8(19,5)$ & $18(12)$ & 0,21 \\
\hline $\begin{array}{l}\text { Dehiscencia } \\
\text { anastomótica }\end{array}$ & $6(16,7)$ & $5(3,4)$ & 0,006 \\
\hline Reoperación & $9(22)$ & $15(10)$ & 0,04 \\
\hline Mortalidad & $2(4,9)$ & 0 & 0,007 \\
\hline Reinternación & $4(9,8)$ & $9(6)$ & 0,40 \\
\hline
\end{tabular}

dos en otros lugares del colon, incluyendo el grupo de pacientes que requirió una colectomía total.

El tiempo operatorio promedio fue de 141,28 minutos 
en el grupo laparoscópico $(\mathrm{DS}=51,65)$ y de $176,34 \mathrm{mi}-$ nutos en el convencional (DS $=85,02$ ), y también hubo diferencias en el número de pacientes a los que se les realizó una anastomosis primaria, siendo este porcentaje mayor en el caso de la cirugía laparoscópica. E1 9,7 \% de los pacientes operados por vía convencional requirieron cuidados intensivos en el postoperatorio. En comparación el grupo laparoscópico que no requirieron de estos cuidados en ningún caso $(\mathrm{p}=0,001)$.

La tabla 3 resume los datos del periodo postoperatorio de los pacientes.

Se encontraron diferencias significativas en los días de internación que requirió cada grupo en promedio, siendo de 13,04 días (DS $=10,10)$ y de 6,95 días $(\mathrm{DS}=4,44)$ para el abordaje convencional y laparoscópico, respectivamente.

La morbilidad de los procedimientos por vía convencional fue significativamente mayor. El $55 \%$ de las complicaciones fueron mayores en este grupo (clasificación de Dindo-Clavien IIIA o mayor), mientras que en el abordaje laparoscópico el 58,4 \% de dichas complicaciones fueron menores (clasificación de Dindo-Clavien I o II).

La dehiscencia global fue del $6 \%$ (11 pacientes presentaron esta complicación sobre un total de 182 pacientes a los que se les realizó anastomosis primaria), siendo más frecuente esta complicación en el grupo convencional. De 6 pacientes que presentaron fístulas en este grupo, 4 presentaron dehiscencias mayores y 2 fueron menores. En el grupo laparoscópico, 3 pacientes sufrieron dehiscencias mayores y 2 menores. La media de edad de los pacientes que presentaron esta complicación fue de 70 años. Cuatro pacientes habían sido sometidos a una cirugía mayor asociada durante el procedimiento.

La tasa de reoperaciones fue significativamente mayor en el grupo convencional, así como también la mortalidad asociada al procedimiento.

La tabla 4 muestra el estadio tumoral de aquellos pacientes operados por adenocarcinoma de colon $(93,2 \%)$. E1 resto de los pacientes fueron intervenidos por tumores malignos de otra estirpe $(6,8 \%)$.

Los pacientes que presentaban estadio 0 de su enfermedad fueron abordados en su totalidad por vía laparoscópica $(p=0,01)$. Por otro lado, aquellos que presentaban estadio IIIC y IVB fueron mayormente abordados por vía convencional ( $p=0,009$ y 0,004 , respectivamente), aunque en ninguno de los 2 grupos se realizó una cirugía asociada para resección de enfermedad oncológica extracolónica. No hubo diferencias en el abordaje en el resto de los estadios que alcanzaran significancia estadística.

\section{DISCUSIÓN}

TABLA 4: ESTADIO TUMORAL

\begin{tabular}{llll} 
Variable & $\begin{array}{l}\text { Cirugía } \\
\text { convencional } \\
\mathrm{n}(\%)\end{array}$ & $\begin{array}{l}\text { Cirugía } \\
\text { laparoscópica } \\
\mathrm{n}(\%)\end{array}$ & Valor de $\mathrm{p}$ \\
$\mathrm{N}$ & 34 & 144 & \\
\hline Estadio 0 & $0(0)$ & $15,3(22)$ & 0,01 \\
\hline Estadio I & $20,6(7)$ & $20,8(30)$ & 0,975 \\
\hline Estadio IIA & $26,5(9)$ & $28,5(41)$ & 0,815 \\
\hline Estadio IIB & $5,9(2)$ & $6,25(9)$ & 0,936 \\
Estadio IIIA & $2,9(1)$ & $8,3(12)$ & 0,277 \\
Estadio IIIB & $8,8(3)$ & $12,5(18)$ & 0,55 \\
\hline Estadio IIIC & $20,6(7)$ & $6,25(9)$ & 0,009 \\
Estadio IVA & $5,9(2)$ & $1,4(2)$ & 0,112 \\
Estadio IVB & $8,8(3)$ & $0,7(1)$ & 0,004
\end{tabular}

La patología maligna del colon requiere de un tratamiento quirúrgico que, si bien originalmente fue descripto por vía abierta, luego de su introducción la vía laparoscópica ha probado ser mejor en términos de menor morbilidad y mortalidad, menor estadía hospitalaria, más rápido retorno a las actividades habituales y con un resultado oncológico equivalente a aquel alcanzado en la cirugía convencional. ${ }^{8-12,19,20}$

Por todo lo dicho, hoy, el abordaje laparoscópico está indicado para el tratamiento de los tumores de colon. Sin embargo, todavía existen grandes problemas para estandarizar el uso de esta técnica, ya sea por dificultad en alcanzar la curva de aprendizaje o por falta de acceso a los materiales necesarios. ${ }^{13,14}$

En nuestra cohorte, con una limitación en cuanto al número de pacientes incluido, hemos encontrado diferencias significativas en términos de morbilidad y mortalidad a favor de la laparoscopía que son consecuentes con aquellas presentadas en otros trabajos que comparan los resultados a corto plazo del abordaje laparoscópico y convencional. ${ }^{8,21}$

Sobre un total de 114 pacientes que presentaban antecedente de cirugías abdominales previas, el $73 \%$ (83 pacientes) fueron operados por vía laparoscópica. Sin embargo, todavía hay un porcentaje de pacientes significativo que fueron intervenidos por vía convencional, lo cual podría indicar que el antecedente de cirugía previa continúa representando una contraindicación relativa para el abordaje laparoscópico. Este comentario también aplica para aquellos pacientes que presentaron tumores en el colon transverso, que fueron abordados en su mayoría por vía abierta.

La vía mínimamente invasiva presentó diferencias significativas en cuanto a tiempo operatorio, requerimientos de cuidados intensivos postoperatorios e índices de morbilidad y mortalidad, incluyendo un mayor índice de complicaciones severas en los pacientes abordados 
por vía abierta (Clavien-Dindo mayor a IIIa). El antecedente de cirugía abdominal previa (mayor en el grupo convencional) podría ser un factor que predisponga a tener más complicaciones. Sin embargo, estadificando los grupos por antecedente de cirugía previa e incidencia de complicaciones, no se encontraron diferencias significativas entre quienes sí habían sido operados previamente $\mathrm{y}$ aquéllos que no $(36 \%$ y $30 \%$, respectivamente, $\mathrm{p}=$ $0,381)$. Tampoco se encontraron diferencias en este aspecto entre grupos que fueron o no operados por recidivas de la enfermedad tumoral.

El porcentaje de pacientes con infección de sitio quirúrgico fue mayor en el grupo abierto, sin embargo, esta diferencia no fue estadísticamente significativa.

$\mathrm{El}$ índice global de dehiscencia anastomótica fue del 6 $\%$ para ambos grupos, resultado que resulta comparable a otros grupos nacionales, ${ }^{22}$ siendo más frecuente esta complicación en el grupo convencional que en el laparoscópico ( 16,7 vs. $3,4 \%, p=0,006)$. A su vez, también presentaron más complicaciones asociadas a requerimiento de reoperaciones y una media de días de internación cercana al doble de aquellos operados por vía laparoscópica. Este es un hallazgo significativo considerando que un argumento en contra de la laparoscopía son los costos asociados al procedimiento (requiere más materiales), pero los mejores resultados disminuyen los costos asociados a la internación y, por ende, al procedimiento en sí. Esto ya ha sido demostrado en otros países. ${ }^{23}$
Un último aspecto que debe ser mencionado en esta discusión es el abordaje de tumores avanzados de colon. En nuestro trabajo, estos tumores fueron operados en su mayoría por vía convencional. Sin embargo, debemos destacar que ya se ha demostrado que estos pacientes pueden ser abordados de forma segura por vía laparoscópica con buenos resultados postoperatorios y oncológicos, ${ }^{24,25}$ y prospectivamente debemos tener esto en consideración para comenzar la cirugía en este tipo de pacientes por vía mínimamente invasiva.

Esta serie es la primera publicada por el servicio de este hospital universitario en cirugía colorrectal laparoscópica y al estar comprendida dentro de la curva de aprendizaje del servicio, el sesgo de selección sigue siendo alto. Esto se ve reflejado en el hecho de que el grupo abierto esté comprendido en gran medida por tumores avanzados y pacientes con antecedentes de cirugía previa. Una futura experiencia seguramente permita reducir aún más el número de pacientes abordados por vía abierta. Otra limitación es el hecho de ser retrospectivo.

\section{AGRADECIMIENTOS}

Agradecimiento al Dr. Juan Carlos Patrón Uriburu por haber colaborado con las correcciones de este trabajo y al Dr. Augusto Carrie y a todo el equipo quirúrgico de $\mathrm{CE}-$ MIC, por haber compartido su casuística de pacientes.

\section{REFERENCIAS}

1. Global cancer observatory. International agency for research on cancer. World Health Organization.

2. Ahmed M. Colon Cancer: A clinician's perspective in 2019. Gastroent Res 2020;13:1-10.

3. Jacobs M, Verdeja JC, Goldstein HS. Minimally invasive colon resection (laparoscopic colectomy). Surg Laparosc Endosc 1991;1:144-50.

4. Monson JR, Darzi A, Carey PD, et al. Prospective evaluation of laparoscopic-assisted colectomy in an unselected group of patients. Lancet 1992;340:831-33.

5. Phillips EH, Franklin M, Carroll BJ, et al. Laparoscopic colectomy. Ann Surg 1992;216:703-7.

6. Berends FJ, Kazemier G, Bonjer HJ, et al. Subcutaneous metastases after laparoscopic colectomy. Lancet 1994;344:58.

7. Johnstone PAS, Rodhe DC, Swartz SE, et al. Port site recurrences after laparoscopic and thoracoscopic procedures in malignancy. J Clin Oncol 1996;14:1950-56.

8. Papageorge CM, Zhao Q, Foley EF, et al. Short-term outcomes of minimally invasive versus open colectomy for colon cancer. J Surg Res 2016;204:83-93.

9. Jayne DG, Guillou PJ, Thorpe H, et al. Randomized trial of laparoscopic-assisted resection of colorectal carcinoma: 3-year results of the UK MRC CLASICC Trial Group. J Clin Oncol 2007;25:3061-68.

10. Fleshman J, Sargent DJ, Green E, et al. Laparoscopic colectomy for cancer is not inferior to open surgery based on 5-year data from the COST Study Group trial. Ann Surg 2007; 246:655-62. discussion

662-54.

11. Buunen M, Veldkamp R, Hop WC, et al. Survival after laparoscopic surgery versus open surgery for colon cancer: long-term outcome of a randomised clinical trial. Lancet Oncol 2009;10:44-52.

12. Kuhry E, Schwenk W, Gaupset R, et al. Long-term outcome of laparoscopic surgery for colorectal cancer: a cochrane systematic review of randomised controlled trials. Cancer Treat Rev 2008;34:498-504.

13. Simorov A, Shaligram A, Shostrom V, et al. Laparoscopic colon resection trends in utilization and rate of conversion to open procedure: a national database review of academic medical centers. Ann Surg 2012;256:462-468.

14. Fox J, Gross CP, Longo W, Reddy V. Laparoscopic colectomy for the treatment of cancer has been widely adopted in the United States. Dis Colon Rectum 2012; 55:501-508.

15. Patrón Uriburu JC, Cillo M, Ruiz H, et al. Adherence to laparoscopic colorectal surgery in Argentina and its relation with the world. Survey results. EC Gastroenterol Dig System 3.5 2017:152-165.

16. Clavien Dindo D, Demartines N, Clavien PA. Classification of surgical complications: A new proposal with evaluation in a cohort of 6336 patients and results of a survey. Ann Surg 2004;240:205-13.

17. Rahbari NN, Weitz J, Hohenberger W, et al. Definition and grading of anastomotic leakage following anterior resection of the rectum: a proposal by the International Study Group of Rectal Cancer. Surgery 2010;147:339-51.

18. American Joint Committee on Cancer (AJCC) TNM staging classification for colon cancer 8th ed. 2017. 
19. Braga M, Vignali A, Gianotti L, et al. Laparoscopic versus open colorectal surgery. A randomized trial on short-term outcome. Ann Surg 2002;236:759-66. disscussion 767.

20. Liang JT, Han KC, Lai HS, et al. Oncologic results of laparoscopic versus conventional open surgery for stage ii or iii left-sided colon cancers: A randomized controlled trial. Ann Surg Oncol 2006;14:109-17.

21. Kennedy GD, Heise C, Rajamanickam V, et al. Laparoscopy decreases postoperative complication rates after abdominal colectomy: results from the national surgical quality improvement program. Ann Surg 2009;249:596-601.

22. Angeramo C, Dreifuss N, Shclottmann F, et al. Postoperative outcomes in patients undergoing colorectal surgery with anastomotic leak before and after hospital discharge. Updates Surg 2020;72:46368.

23. Sajid MS, Rathore MA, Baig MK, et al. A critical appraisal of the cost effectiveness of laparoscopic colorectal surgery for oncological and non-oncological resections. Updates Surg 2017;69:339-44.

24. Klaver CEL, Kappen TM, Borstlap WAA, et al. Laparoscopic surgery for T4 colon cancer: a systematic review and meta-analysis. Surg Endosc 2017;31:4902-12.

25. Kim IY, Kim BR, K HS, et al. Differences in clinical features between laparoscopy and open resection for primary tumor in patients with stage IV colorectal cancer. Onco Targets Ther 2015;8:3441-48. 